\title{
Integrated systems for rapid point of care (PoC) blood cell analysis $\uparrow$
}

\author{
Cees van Berkel, ${ }^{a}$ James D. Gwyer, ${ }^{a}$ Steve Deane, ${ }^{a}$ Nicolas Green, ${ }^{b}$ Judith Holloway, ${ }^{c}$ Veronica Hollis ${ }^{b}$ \\ and Hywel Morgan*b
}

\author{
Received 11th November 2010, Accepted 20th January 2011 \\ DOI: 10.1039/c0lc00587h
}

Counting the different subpopulations of cells in a fingerprick of human blood is important for a number of clinical point-of-care $(\mathrm{PoC})$ applications. It is a challenge to demonstrate the integration of sample preparation and detection techniques in a single platform. In this paper we demonstrate a generic microfluidic platform that combines sample processing and characterisation and enumeration in a single, integrated system. Results of microfluidic 3-part differential leukocyte (granulocyte, lymphocyte and monocyte) counts, together with erythrocyte and thrombocyte (platelet) counts, in human blood are shown and corroborated with results from hospital clinical laboratory analysis.

\section{Introduction}

\subsection{Point-of-care haematology}

Diagnostic testing at or near the site of patient care is often termed 'near-patient' or 'point-of-care' (PoC) testing. The applications of $\mathrm{PoC}$ testing are widespread, including the use of instrument-free, disposable reagent test strips, such as the home pregnancy kit or blood glucose test, through to portable handheld or bench-top devices used, for example, in blood gas analysis (see Price ${ }^{1}$ for a review).

In recent years, PoC testing in haematology has increased significantly. The full blood count (FBC), the most common clinical indicator of patient health, is one area where $\mathrm{PoC}$ systems are expected to make a significant impact. The FBC includes (at least) a 3- (or 5-)part leukocyte, or white blood cell (WBC) differential count, haemoglobin $(\mathrm{Hb})$ concentration, platelet (PLT) count, red blood cell (RBC) count and a variety of red cell indices related to red cell size and haemoglobin content.

ICSH guidelines ${ }^{2}$ have identified a clear need for a PoC FBC for use in areas including GP surgeries, pharmacies and residential settings. Such a device needs to be portable, the test must be minimally invasive and the system simple to use. For selftesting devices, capillary blood can be collected from a finger puncture, such as with glucose tests ${ }^{3}$ or anticoagulation therapy. ${ }^{4}$

There are some examples of small FBC systems. The simplest is the HemoCue WBC system, ${ }^{5,6}$ which requires $10 \mu \mathrm{L}$ of blood

${ }^{a}$ Philips Research Laboratories, 101 Cambridge Science Park, Milton Road, Cambridge, CB4 OFY, UK

${ }^{b}$ School of Electronics and Computing Science, University of Southampton, Highfield, Southampton, SO171BJ, UK.E-mail: hm@ecs.soton.ac.uk; Fax: +44(0)2380 593029; Tel: +44(0)2380 593330

${ }^{c}$ Division of Infection, Inflammation and Repair, School of Medicine, University of Southampton, Southampton, SO16 6 YD, UK

$\uparrow$ Published as part of a LOC themed issue dedicated to UK Research: Guest Editors Professors Hywel Morgan and Andrew deMello. and measures total WBC count (no differential). The Chempaq device uses a disposable cartridge that is filled with $20 \mu \mathrm{L}$ of blood. It measures a 3 part differential WBC, RBC and platelet counts with a single disposable cartridge. ${ }^{7-9}$ All these devices have been CE marked (Europe) and 510k approved (US), which allows their use in the clinical environment by trained professionals. They are not, however, approved for 'self-testing' use by the patient.

\subsection{Sample preparation: microfluidics}

A small $2 \mu \mathrm{L}$ drop of blood contains 10000000 erythrocytes and 14000 leukocytes of which only 600 will be monocytes. To cover this very large dynamic range, the sample is used with dilution ratios of $1: 10000$ or $1: 20$ for erythrocytes and leukocytes, respectively. To count all the available monocytes within for example one minute implies flow rates of at least $40 \mu \mathrm{L} \mathrm{min}{ }^{-1}$; microfluidic technologies offer solutions to handling and processing such volumes. ${ }^{10,11}$ The HemoCue cuvette is one example of a haematology test that uses a very simple microfluidic cartridge. In this system, $10 \mu \mathrm{L}$ of blood is drawn by capillary forces into a disposable micro-cuvette containing the dried reagents necessary for erythrocyte lysis and either haemoglobin labelling or WBC staining. ${ }^{5}$

The demand for complex multi-parameter analysis of blood necessitates a different approach to sample preparation. In millifluidic approaches, ${ }^{7,12}$ such as the Chempaq cartridge, the blood is mixed in a reservoir after capillary metering of a known volume of blood. Reagents are stored in the mixing reservoir as liquids and convective mixing is achieved by a small magnetic stirrer. Pumping relies on externally delivered gas pressure.

Networks of microfluidic channels can be designed and tuned to achieve desired mixing and dilution ratios while minimizing the need for externally driven valves or pumps. ${ }^{13,14}$ Microfluidic devices also offer methods for controlled cell lysis, and a device for rapid and continuous erythrocyte lysis has been reported. ${ }^{15}$ 
The lysis reagent sheathes a stream of whole blood, lysing the RBCs. Following lysis, cells are returned to isotonic conditions by addition of phosphate buffered saline (PBS).

\subsection{Sample detection: impedance cytometry}

Cells are usually identified and enumerated using optical (light scatter) or electrical (Coulter) methods, or a combination of both. Electrical detection methods readily lend themselves to miniaturisation. The first cytometer capable of counting and sizing single particles was developed by Coulter. ${ }^{17}$ It measures the DC resistance (or low frequency impedance) change caused by a cell passing through a narrow orifice separating two electrically isolated fluid-filled chambers. This principle has been miniaturised in the form of a micro-Coulter counter by a number of authors. ${ }^{18}$ Microfluidic impedance cytometry (MIC) is an evolution of the micro-Coulter counter where micro-electrodes are integrated into the walls of the micro-channel and cell impedance properties are measured over a range of frequencies. Recently Holmes et al. $^{19}$ demonstrated the use of MIC for identification and enumeration of the three common leukocytes in human blood. Further developments include the use of an "impedance label" to aid in discriminating sub-populations of cells, such as CD4+ monocytes in a mixed population. ${ }^{20}$ For recent reviews on the technology see Sun and Morgan ${ }^{18}$ and Cheung et al. $^{21}$

The principal distinction between micro-Coulter counters and MIC is the fact that in the former the electric field is in the same direction (longitudinal) to the fluid flow and confined by the diameter and length of the orifice, while in the latter the electric field is substantially perpendicular to the fluid flow and confined by the diameter of the channel and the geometry of the electrodes. The two approaches deal in a fundamentally different way with the unavoidable issue of electrolysis. In micro-Coulter counters the electrodes tend to be large and well removed from the orifice to spread bubble formation over a large (electrode) area and keep the gas products away from the orifice. In contrast, in MIC the electrodes are small and placed in the high flow region of the channel itself which means that products of electrolysis are continuously removed by the flow. This means that MIC can be easily integrated with the continuous-flow sample preparation techniques discussed in the previous section. In addition, the confinement of the electric field means that in MIC it is easy to incorporate pairs of electrodes, so that differential sensing can be used to correct for any drift in the electrode-fluid interface, or the properties of the carrier fluid. Moreover, because the electrodes are within the channel itself, there are no aperture access resistances and the sensing volume itself is small, leading to an improvement of thermal noise limit of roughly a factor of 2, which in turn leads to a larger dynamic range of particles for a given aperture. The small parasitic capacitances associated with microelectrode systems also allow measurements to be made at relatively high frequencies.

In this paper we describe the design and testing of a generic microfluidic platform technology which combines sample preparation with impedance cytometry in a single, hybrid cartridge. The system takes a small volume of human blood, provides all sample preparation and measures a 3-part differential, platelet and erythrocyte counts and haemoglobin concentration.

\section{Microfluidic impedance cytometry for FBC}

The FBC system consists of two parts, the impedance measuring chip (shown schematically in Fig. 1) and a microfluidic sample preparation block (Fig. 2). Full details of the impedance chip shown in Fig. 1 have been described previously. ${ }^{19}$ Briefly, two pairs of electrodes are fabricated on the top and bottom of a micro-channel made from thick resist on glass substrates. The measured signal is the electrical impedance (real and imaginary) at suitable applied frequencies (typically two). The signal transient provides information on cell size, shape, membrane capacitance, velocity and position in the channel.

A schematic diagram of the microfluidic sample preparation block is shown in Fig. 2a. The microfluidic block performs sample (whole blood) loading, pre-treatment and dilution into two separate fluid channels followed by two separate impedance chips that are bonded to the block. The bottom arm (containing ports 1 and 2) takes part of a single blood sample and performs analysis of $\mathrm{WBC}$ differential and $\mathrm{Hb}$ concentration. The upper arm (containing port 3) performs RBC and platelet (PLT) count. The microfluidic system was designed under the assumption of low Reynolds numbers which simplifies the design and allows the microfluidic system to be modelled as a resistive network with flow rate equivalent to current and pressure equivalent to potential. The simulation was performed using a PSpice-based simulator. Fig. 2b shows a CAD layout of the final microfluidic circuit that is used to process the blood, with separate WBC and RBC/PLT analysis areas. The microfluidic channels are $200 \mu \mathrm{m}$ $\times 100 \mu \mathrm{m}$ and the flows are controlled by varying the length (resistance) of the different sections. The two optical windows are used for $\mathrm{Hb}$ measurement, determined using light absorbance. The various inputs are connected to syringe pumps of different volume driven by a common mechanism. In future these syringe pumps will be integrated into a single disposable cartridge. A photograph of the final device is shown in Fig. 2c.

\subsection{WBC channel}

For WBC analysis, the erythrocytes are lysed in continuous flow using a mixture of detergent and formic acid. ${ }^{19,23}$ The lysis
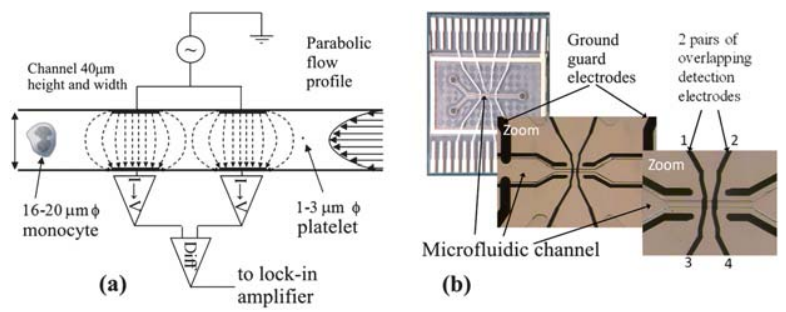

(b)

Fig. 1 (a) Schematic diagram of the microfluidic impedance sensor, and the configuration of the detection electronics. The channel is a square cross section $(40 \mu \mathrm{m} \times 40 \mu \mathrm{m})$, and the electrodes are $30 \mu \mathrm{m}$ wide with a $40 \mu \mathrm{m}$ gap. The figure shows the largest common blood cell (monocytes, $\sim 16$ to $20 \mu \mathrm{m}$ diameter) and the platelet size $(1-3 \mu \mathrm{m}$ diameter $)$ for reference. (b) Photographs of the impedance chip, which is $15 \mathrm{~mm} \times$ $20 \mathrm{~mm}$ in size, with two zooms of the sensing region. This consists of two pairs of overlapping electrodes; signal input is on the top pair $(1,2)$; the bottom pair $(3,4)$ is connected to a differential virtual ground current sensing electronics. 

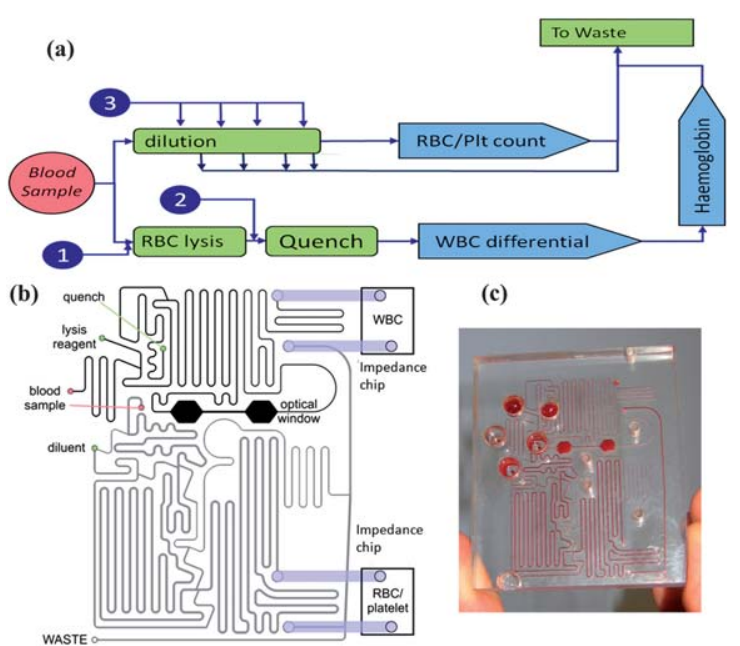

Fig. 2 (a) Diagram of the WBC/RBC chip processes incorporating lysis and quench of the blood, followed by WBC count (with optical windows for haemoglobin). Dilution of the blood for RBC/PLT analysis is shown in the upper arm. (b) CAD drawing of the microfluidic block showing the different length channels, the input ports and the two impedance chips. (c) Photograph of the final microfluidic device made in PMMA.

reagent is delivered by a syringe pump to port 1 where it mixes with the blood sample that is pumped using a second syringe pump at a constant flow rate. The initial width of the blood stream (in the $200 \mu \mathrm{m}$ wide channel) is $13 \mu \mathrm{m}$ and diffusion of the lysis reagent into the blood stream happens quickly $(<1 \mathrm{~s})$. The distance between ports 1 and 2 in Fig. 2a provides 6 seconds exposure of sample to lysis reagent. The lysis chemistry is quenched using a second reagent delivered to port 2. The second longer channel (following port 2) mixes the quench solution with the lysate, neutralising and stabilising the chemistry. This produces a clean substantially debris-free sample, containing leukocytes, which are subsequently analysed by the impedance chip.

\subsection{RBC/platelet (PLT) channel}

For RBC and PLT analysis, a small amount of the same blood sample is pumped through the top arm of the device (Fig. 2a), where it is mixed with a diluent that is pumped into port 3 at four separate locations. At each point the sample is serially diluted, to give a 10000 -fold total dilution. At each stage, the blood and diluent passes through a long mixing channel before being separated into two; the largest volume is sent to waste, the smaller to the next dilution stage. The channel lengths are designed to allow sufficient mixing time, to balance the flow rates of the different fluids and to match the impedance of the RBC line to that of the WBC line.

\subsection{Junction design}

For the successful design and stable operation of the microfluidic sample preparation it is important that the behaviour of the system is linear over the flow rates of interest. The Reynolds number $(R e)$ is the ratio of the size of the inertial force to the viscous forces on a fluid element:

$$
R e=\frac{\rho}{\eta} u D_{\mathrm{h}}=\frac{\rho}{\eta} u d
$$

where $\rho$ fluid density, $u$ the average velocity of the fluid, $\eta$ is the viscosity of the fluid and $D_{\mathrm{h}}$ the hydraulic diameter of the channel, which for a square channel is its width $d$. Recasting this in terms of flow rate $Q=u d^{2}$ gives

$$
R e=\frac{\rho}{\eta} \frac{u d^{2}}{d}=\frac{\rho}{\eta} \frac{Q}{d}
$$

This equation shows that for a required flow rate, a smaller channel $(d)$ requires a larger pressure but leads to an increase in the Reynolds number. Typical values for our system are $Q=40$

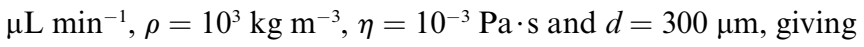
$R e=2.22$.

This moderate Reynolds number indicates that the inertial forces are strong and care needs to be taken in the design of the microfluidic sample preparation to ensure linear and stable operation. In particular the behaviour of the microfluidic junctions should be independent of flow rate up to moderate Reynolds number. To validate this, the behaviour of the junctions was modelled for different Reynolds number. RBC measurement requires sequential dilution of the sample, typically performed using a T-shaped junction shown in Fig. 3a. The sample is first divided, and most of the material goes to waste, and a fraction (in the case of these simulations and one junction in the device, a fraction of 0.1 ) passes to the second junction where it is mixed with the diluent. The behaviour of this junction was simulated for a wide range of Reynolds number and the siphon ratio, defined as the fraction of the sample passing the first junction to be diluted, was calculated and is shown in Fig. 3d. The flow was simulated using FlexPDE (TM, PDE Solutions, US) for the channel sizes used in the physical device: $300 \mu \mathrm{m}$ for the sample channel and $200 \mu \mathrm{m}$ for the waste and diluent channels. The junctions were located in a long simulation domain with straight channels and the flow rates were modified (a)

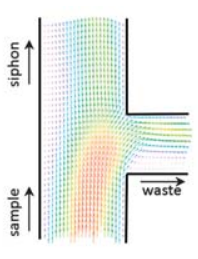

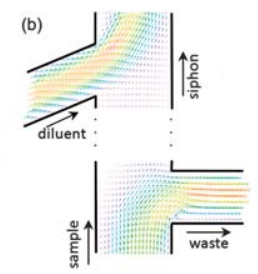

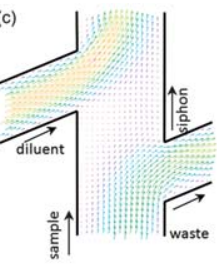

(d)

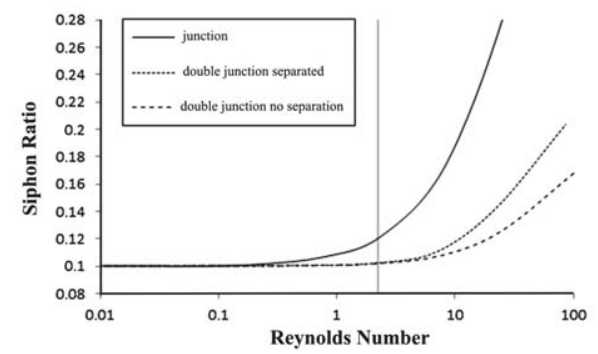

Fig. 3 Simulations of flow profiles in microfluidic junction of different designs at $R e=2.2$. (a) Single $\mathrm{T}$ junction. (b) Separated double junction. (c) Optimally spaced double junction. (d) Siphon ratio as function of Reynolds number for the different designs. 
by controlling pressure over a wide range applied to inputs and outputs.

As can be seen from the figure, the siphon ratio depends strongly on flow rate (Reynolds number) with linear flow and fixed ratio up to a Reynolds number of approximately 0.2 and an increasing ratio for higher flow rates. This simple design causes large errors in dilution at the minimum flow rates required by our application as indicated by the vertical grey line in Fig. 3d. Optimisation of channel angles to accommodate the inertial forces at the first and second junction, as illustrated in Fig. 3b, stabilises the flow to an extent. Decreasing the separation distance between the junctions as shown in Fig. 3c further improves the potential range of operation. The behaviour is dependent on the balance of mass flows, i.e. that the waste flow rate and the diluent flow rate are the same.

If these are different, then the range of linear behaviour decreases. This implies that when the Reynolds number is high, the inertial effects can be mitigated by designing the junctions in such a way that the inertias of the different flows balance each other, demonstrated by the improved operation when the two junctions are brought together. This junction design (or similar) guarantees that the simulation of the entire microfluidic system is linear and can be modelled using equivalent circuit analysis.

\subsection{Signal analysis and processing}

The largest cells in whole blood are monocytes, with typical diameters in the region of $20 \mu \mathrm{m}(\sim 4000 \mathrm{fL})$. Because it is important to avoid clogging in the device, the channel width and height in the impedance analysis chips were set to $40 \mu \mathrm{m}$ (see Fig. 1). This implies a sensing volume of $64000 \mathrm{fL}$. Although this is 3 to 5 times less than the sensing volume of a comparable Coulter aperture, the measurement of small objects like platelets remains challenging. Mean platelet volumes are typically $6 \mathrm{fL}$ and a reliable count requires a detection limit of $1 \mathrm{fL}$, i.e. a measurement of approximately $0.002 \%$ volume fraction.

Fast diagnosis requires fast sample processing and analysis. To a first approximation a $2 \mu \mathrm{L}$ blood sample should be measured in approximately 1 minute. Therefore, the velocity of the fastest cells (midstream in the flow) is $\sim 1 \mathrm{~m} \mathrm{~s}^{-1}$, implying a minimum event of $\sim 0.1 \mathrm{~ms}$ (for the electrode gap used in these devices). The bandwidth of the detection electronics must be sufficiently wide to detect these fast moving events without distortion. The overall noise level in the electronics is determined by the thermal noise in the channel impedance and the current-to-voltage converter feedback resistors, which sets a fundamental limit. Thermal noise increases as the square root of the system bandwidth, therefore the noise level increases by a factor of 2 when the cell speed is increased by a factor of 4 . The signal level remains constant so the signal-to-noise ratio deteriorates by a factor of 2 . In order to run at such high speeds, custom lock-in amplifiers were designed with optimised 5-pole band pass Bessel filters. The Bessel characteristic preserves the pulse shape without undue distortion, and the band pass was chosen to allow up to the $5^{\text {th }}$ harmonic component of the fastest events to pass. With a maximum cell speed of $\sim 1 \mathrm{~m} \mathrm{~s}^{-1}$, and with the $70 \mu \mathrm{m}$ centre-to-centre distance of the electrodes, this gives a maximum fundamental frequency of the cell events in the region of $7 \mathrm{kHz}$, and a $5^{\text {th }}$ harmonic of $35 \mathrm{kHz}$.
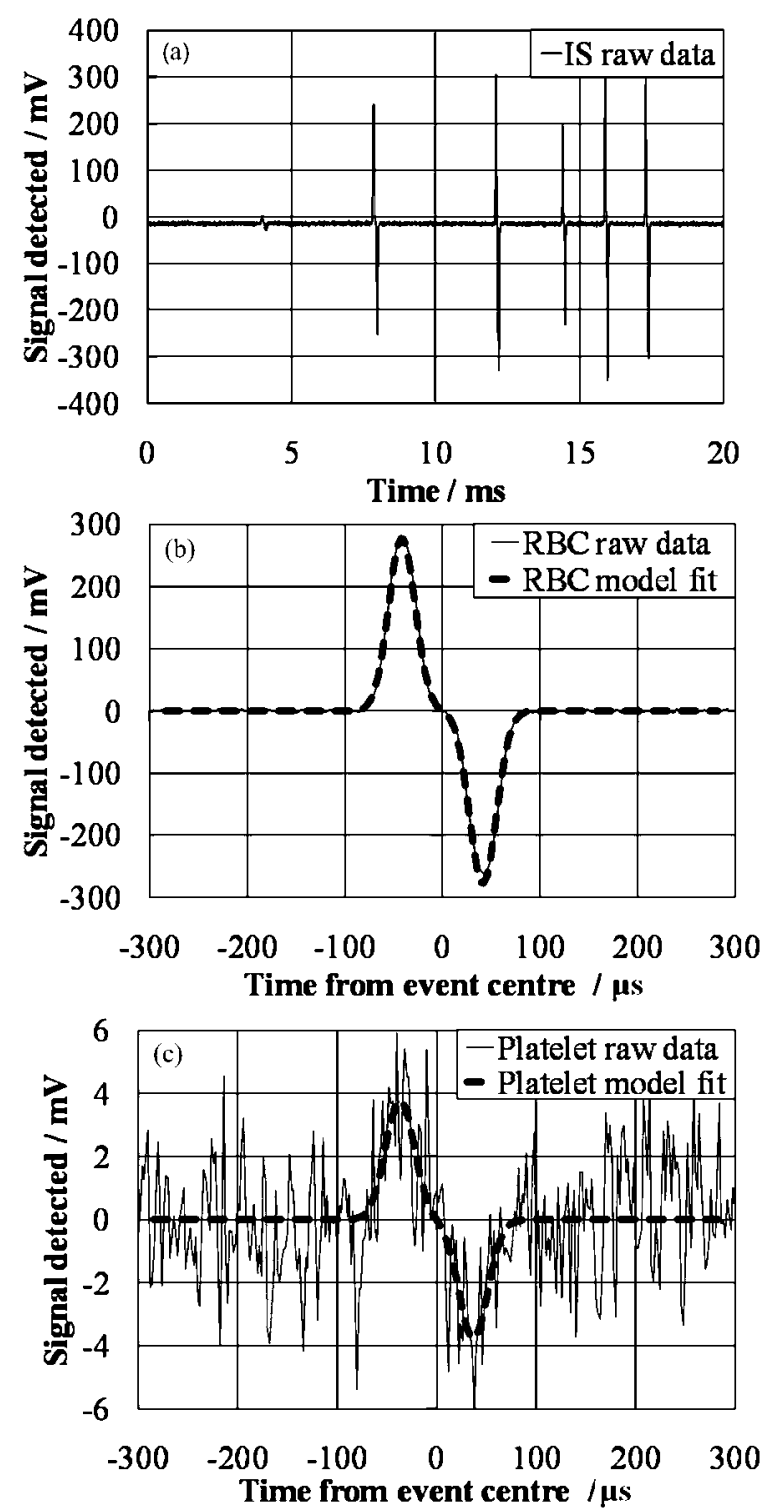

Fig. 4 (a) Time trace of the unprocessed data captured by the impedance system. The voltage signal is the magnitude of the impedance measured at a single frequency. (b) Voltage from a single red blood cell (RBC) $(\sim 70$ $\mu \mathrm{m}^{3}$ ) event. The raw data and fitted data are shown. (c) Similar signal for a small $\left(\sim 1 \mu \mathrm{m}^{3}\right)$ platelet, with the raw data and fitted model.

Fig. 4 shows typical data captured by the system. Fig. 4a illustrates a $20 \mathrm{~ms}$ time frame for a sample of diluted whole blood. The trace shows a small particle followed by 5 larger particles. Note that dilution of the sample ensures that most particles arrive separately at random intervals, and overlapping events are relatively rare $(<5 \%)$. Individual events are recovered from the data by matching it against a model function ${ }^{24}$ of a pair of anti-symmetric Gaussian peaks. This is illustrated in Fig. 4b, which provides a close up of a red blood cell $(\mathrm{RBC})(\sim 70 \mathrm{fL})$ event and the model function. In this the correlation coefficient between the data and model function is $>98 \%$. Fig. $4 \mathrm{c}$ shows the data and fit for a small ( $\sim 1 \mathrm{fL})$ platelet event. This illustrates that the use of a model function can reliably extract events even when they are comparable to the thermal noise level. ${ }^{24}$ The 
experimental relationship between impedance measurements and cell volume was made through reference experiments with beads. ${ }^{19}$ For particles which are small $(<500 \mathrm{fL})$ compared to the measurement volume (64000 fL), the volume scale is approximately linear, while for larger particles it is sub-linear. ${ }^{\mathbf{1 8 , 2 2}}$ For this particular system, the signal is $\sim 7 \mathrm{mV} \mathrm{fL}^{-1}$ for particles the size of red blood cells or smaller.

\section{Experimental}

The impedance chips were fabricated from pairs of glass substrates bonded using adhesive bonding methods. The channel walls are fabricated from SU-8 and the Pt electrodes manufactured using conventional evaporation and photolithography techniques. The microfluidic cartridges are micro-milled in PMMA (Carville, UK). Whole venous blood was collected into EDTA coated vacutainers from donors participating in the Philips Research UK donation programme. Lysis solution was $0.12 \% \mathrm{v} / \mathrm{v}$ formic acid, $0.05 \% \mathrm{w} / \mathrm{v}$ saponin; quench solution was $0.6 \% \mathrm{w} / \mathrm{v}$ sodium carbonate, $3 \% \mathrm{w} / \mathrm{v}$ sodium chloride. The RBC diluent was pocH-pack D diluent (Sysmex, Japan). Blood was obtained from Addenbrooke's Hospital pathology laboratory and University College London Hospital and accompanied by reference data. Further experimental details can be found elsewhere. ${ }^{19}$

\section{Results}

\subsection{Microfluidic lysis WBC}

An example of a WBC differential is given in Fig. 5a, which shows an impedance scatter plot for lysed whole blood, where the lysis was performed in bulk. ${ }^{19}$ The $x$-axis is the impedance magnitude measured at $500 \mathrm{kHz}$, which is a measure of the "electrical" volume of the cells $(\Phi)$. The $y$-axis is the ratio of impedance magnitude measured at $1.7 \mathrm{MHz}$ to $0.5 \mathrm{MHz}$, termed the opacity $(O)$. This parameter measures membrane properties (capacitance) normalised for cell size. The three main sub-
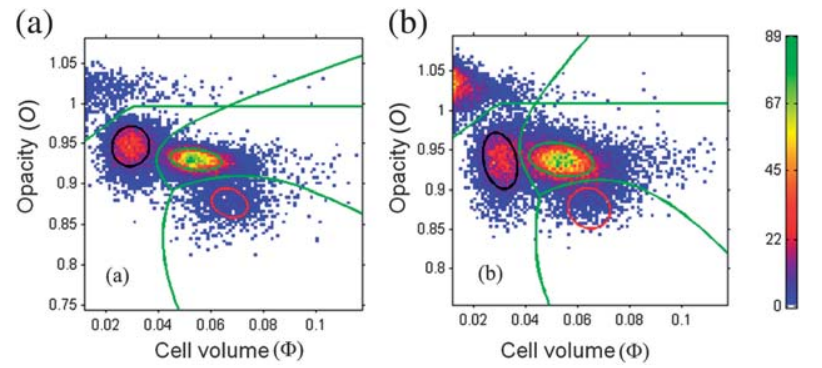

Fig. 5 Impedance scatter plots for WBC after lysis colour coded according to the number of cells. The scatter plot shows cell membrane opacity $(O)$ - the ratio of impedance measured at $1.7 \mathrm{MHz}$ to $0.5 \mathrm{MHz}$ plotted against the (electrical) cell volume $(\Phi)$, measured by the impedance magnitude at $500 \mathrm{kHz}$. (a) Data for whole blood, lysed in bulk. (b) Data for whole blood lysed using the microfluidic network of Fig. 2. Ellipsoids are fits determined from. The fitted populations of the 2D Gaussian are illustrated by the three ovals. The green lines mark positions of equal probability deviation between the different populations. They provide the gating boundaries to obtain an accurate count for lymphocytes, granulocytes and monocytes. populations (lymphocytes, monocytes and neutrophils) are clearly visible (density plot is colour coded). The events at the top left are RBC ghosts and other debris that is not completely eliminated by the lysis. Fig. $5 b$ shows data for the same sample lysed using the microfluidic system. The data are very similar to the bulk lysis sample, except that the ghost/debris content is higher.

In the opacity/volume diagram the experimental results of all cells are summarised by a distribution function $N(\Phi, O)$. We assume that this distribution function is made up of the sum of individual cell events of different types (lymphocytes, monocytes and neutrophils) which are distributed normally around certain means. For example, for the lymphocytes

$$
\begin{aligned}
P_{\mathrm{L}}(\Phi, O)= & \frac{1}{2 \pi \sigma_{1} \sigma_{2}} \exp \left[-\frac{\left(\left(\Phi-\Phi_{\mathrm{L}}\right) \cos \theta+\left(O-O_{\mathrm{L}}\right) \sin \theta\right)^{2}}{2 \sigma_{1}^{2}}\right. \\
& \left.-\frac{\left(\left(O-O_{\mathrm{L}}\right) \cos \theta+\left(\Phi-\Phi_{\mathrm{L}}\right) \sin \theta\right)^{2}}{2 \sigma_{2}^{2}}\right]
\end{aligned}
$$

where $\Phi_{\mathrm{L}}$ and $O_{\mathrm{L}}$ are mean lymphocyte volume and opacity, and $\sigma_{1}, \sigma_{2}, \theta$ are parameters that describe the spread and orientation of the ellipsoidal multi-variate distribution. Similar expression can be written for $P_{\mathrm{G}}(\Phi, O)$ and $P_{\mathrm{M}}(\Phi, O)$, the granulocyte and monocyte populations respectively. In Fig. 5, the fitted populations of the 2D Gaussian are illustrated by the three ovals. The correlation coefficient for this fit and the original data is $97 \%$ for Fig. 5a and 96\% for Fig. 5b, indicating that the Gaussian distributions provide a good description of the measured data. The green lines in Fig. 5 mark positions of equal probability deviation between the different populations. They provide the gating boundaries to obtain an accurate count for lymphocytes, granulocytes and monocytes. The process of 2D Gaussian fitting, gating and counting is fully automated.

Table 1 shows the cell counts obtained in this way as relative cell populations for both the bulk lysis experiments of Fig. 5a and microfluidic lysis experiments of Fig. 5b. The cell counts obtained for the same sample, measured in the hospital lab (Addenbrooke's Hospital, Cambridge, UK), are also shown. As can been seen, good agreement exists between the different results. Previously we demonstrated good concordance between MIC measurements and hospital data for a range of blood samples measured with bulk lysis. ${ }^{19}$ Similar concordance is seen for the microfluidic lysis, with correlation factors of $>97 \%$.

\subsection{RBC/PLT}

Counting of RBCs and PLTs was performed using a single frequency $(500 \mathrm{kHz})$. The cells are easily differentiated by size,

Table 1 Relative white blood cell counts for the results in Fig. 5

\begin{tabular}{llll}
\hline & $\begin{array}{l}\text { Lymphocytes } \\
(\%)\end{array}$ & $\begin{array}{l}\text { Monocytes } \\
(\%)\end{array}$ & $\begin{array}{l}\text { Granulocytes } \\
(\%)\end{array}$ \\
\hline Hospital & 32 & 7 & 61 \\
Bulk lysis + MIC & 34 & 8 & 58 \\
Microfluidic lysis + & 27 & 8 & 65 \\
$\quad$ MIC & & & \\
\hline
\end{tabular}


simplifying the analysis. A representative dataset for a $1: 10000$ diluted blood sample is shown in Fig. 6. Fig. 6a shows a frequency histogram of events plotted on a logarithmic scale. The RBC population is approximately 100 times greater than the PLT, and the scales are adjusted accordingly. Both the RBC and PLT size distribution have a characteristic log-normal distribution. The peak in the platelet distribution corresponds to the mean human platelet volume ${ }^{25}$ of $6 \mathrm{fL}$. In absolute numbers there are 2570 RBCs per $n L$ and 147 platelets per $n L$. Fig. 6 b shows an intensity plot of events, where the velocity of the particles (impedance transient time) is plotted against $\log _{10}(\Phi)$. The RBC intensity distribution shows a peak near $0.8 \mathrm{~m} \mathrm{~s}^{-1}$, with a maximum velocity near $1.1 \mathrm{~m} \mathrm{~s}^{-1}$. At these high flow rates, the RBCs are likely to focus into a preferential position in the channel, ${ }^{16}$ which results in the distribution shown in the figure. There is no evidence that the platelet intensity distribution is other than random, indicating that these much smaller particles do not focus (the lift forces are too small). Fig. $6 \mathrm{~b}$ shows better separation between platelets and RBCs than is evident from Fig. 6a. The aggregate frequency histogram for both platelets and RBCs at different velocities is shown in Fig. 6c. Fig. 6d shows an intensity plot of the correlation coefficient, or goodness of fit, of each event against the model function of anti-symmetric Gaussian peaks. ${ }^{24}$

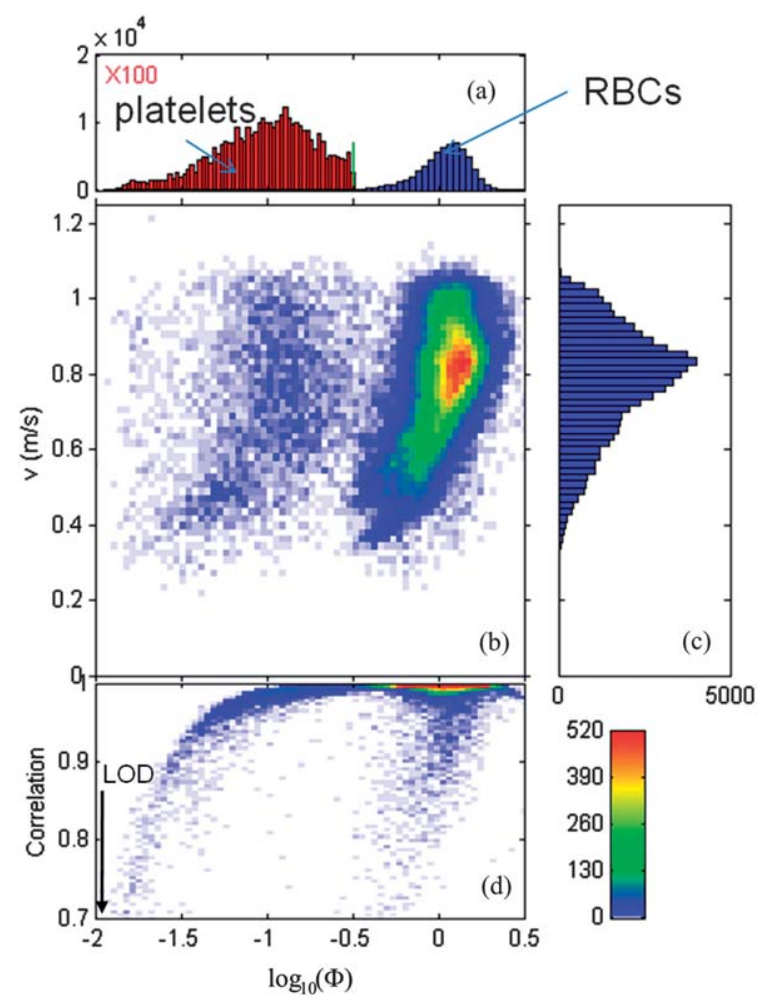

Fig. 6 Red blood cell and platelet data. (a) Event size histogram for RBC and PLT. There are approximately 100 times fewer platelets events than RBCs and the frequency axis is scaled by $\times 100$. (b) Intensity plot of individual events plotted as function of size and velocity. (c) Velocity histogram. (d) Intensity plot of individual events plotted as function correlation coefficient and size. The limit of detection (LOD) is indicated by the arrow (left-hand side of figure).

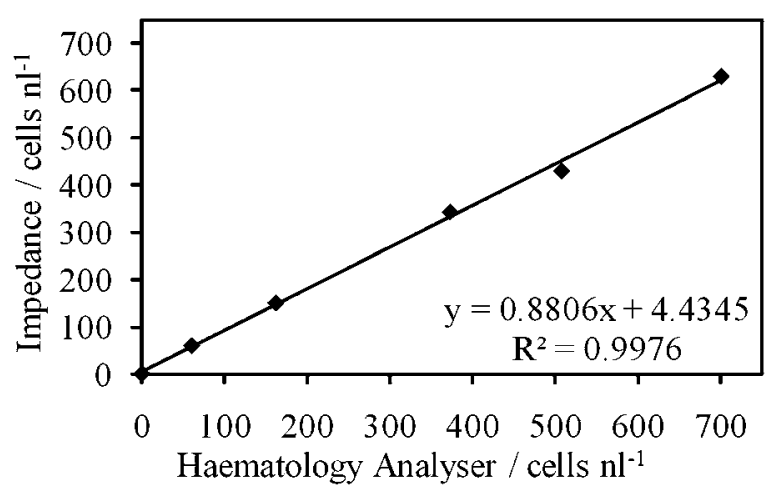

Fig. 7 Platelet data from clinically sourced, diluted human whole blood samples, showing excellent linearity between the absolute platelet counts obtained from the impedance cytometry system and the hospital central lab haematology analyzer.

The intensity colour scale is the same as in Fig. $6 \mathrm{~b}$, and the vast majority of individual events are fitted with a correlation coefficient of better than $98 \%$. The tail with lower correlation coefficients of RBC sized events is due to cells that have some overlap with other cells in the measurement volume. The roll-off in correlation on the left hand side is signals from very small events that approach the signal noise floor. The vertical arrow in Fig. 6d indicates the limit of detection (LOD) of the current system at $70 \%$ correlation coefficient. The LOD is approximately an order of magnitude below the mean platelet volume, giving a value of approximately $0.6 \mathrm{fL}$ or $1 \mu \mathrm{m}$ diameter. This LOD means that the platelets are sized accurately over the entire distribution. The deviation from the expected log-normal distribution may be due to cellular debris ${ }^{26}$ or may indicate that the system can identify other smaller objects such as micro-particles. ${ }^{27}$

\subsection{Platelet concordance}

Concordance between PLT counts, especially for very low platelet numbers is an important challenge for a haematology PoC test. Data for clinical whole blood samples are shown in Fig. 7. Comparison of absolute platelet counts with a standard haematology analyzer revealed excellent linearity with $R^{2}=$ $99.8 \%$, and an offset of 4 platelets $\mathrm{nL}^{-1}$. Blank control runs showed a background count of $(2.9 \pm 0.3)$ platelets per $n L$. The small offset indicates that the diluent was largely free of plateletsized debris, and that the ability to discriminate platelets from noise is good. However, the fit revealed under-counting of the platelets by $12 \%$ relative to the reference method. A similar undercounting was also observed for the RBCs. The contributing main cause of this systematic error is coincident events over the impedance electrodes that cannot be separated. Straightforward stochastic reasoning indicates that this effect accounts for $3-5 \%$ undercounting. As the correlation coefficient is so high, we speculate that the remaining undercounting is due to a systematic error in the dilution process.

\section{Conclusions and outlook}

Identifying and counting the different subpopulations of human blood cells are both clinically useful and technologically 
challenging. It provides a useful example application for the creation of a generic microfluidic platform technology in a single, hybrid cartridge. The principal demand is to combine sample preparation with impedance cytometry to cover the large dynamic range in cell number, size and morphology. In particular, the ability to count both the relatively abundant, but small platelets at the same time as counting the relatively rare, but large monocytes, is a challenge. The technologies require good control of chemistry, accurate timing and control of flow, and precise control of concentrations, all of which are delivered through a well designed microfluidic sample preparation. The system described in this paper performed cell analysis and enumeration coupled with sample pre-processing and dilution. Using human blood samples from clinical sources, it has demonstrated good concordance with large scale hospital analysers. Beyond PoC blood cell counting, the microfluidic platform technology will be useful in a range of applications in the future, including subtyping of blood cells, counting rare cells, cell assays and other matrices.

\section{Acknowledgements}

The authors acknowledge the Technology Strategy Board and EPSRC for funding this work. We also acknowledge Katie Chamberlain for fabricating the impedance chips.

\section{References}

1 C. P. Price, Br. Med. J., 2001, 322, 1285-1288.

2 C. Briggs, J. Carter, S.-H. Lee, L. Sandhaus, R. Simon-Lopez and J.L. Vives Corrons, Int. J. Lab. Hematol., 2008, 30, 105-116.

3 M. Montagnana, M. Caputo, D. Giavarina and G. Lippi, Clin. Chim. Acta, 2009, 402, 7-13.

4 S. Braun, M. Spannagl and H. Völler, Anal. Bioanal. Chem., 2009, 393, 1463-1471.

5 A. Osei-Bimpong, C. Jury, R. McLean and S. M. Lewis, Int. J. Lab. Hematol., 2008, 31, 657-664.
6 J. R. Casey and M. E. Pichichero, Clin. Pediatr. (N. Y., NY, U. S.), 2009, 48, 291-294.

7 B. A. Ekberg, U. D. Larsen and N. Fogh-Andersen, Point Care, 2005, 4, 64-65.

8 L. V. Rao, B. A. Ekberg, D. Connor, F. Jakubiak, G. M. Vallaro and M. Snyder, Clin. Chim. Acta, 2008, 389, 120-125.

$9 \mathrm{http}: / /$ www.Chempaq.com.

10 M. Toner and D. Irimia, Annu. Rev. Biomed. Eng., 2005, 7, 77-103.

11 D. Heikali and D. Di Carlo, JALA, 2010, 15, 319-328.

12 T. Takubo, N. Tsuchiya, K. Miyamura, Y. Sugiyama, I. Tsuda and M. Miyazaki, Point Care, 2007, 6, 174-175.

13 C. Kim, K. Lee, J. H. Kim, K. S. Shin, K. Lee, T. S. Kim and J. Y. Kang, Lab Chip, 2008, 8, 473-479.

14 K. Hattori, S. Sugiura and T. Kanamori, Lab Chip, 2009, 9, 1763 1772.

15 P. Sethu, M. Anahtar, L. L. Moldawer, R. G. Tompkins and M. Toner, Anal. Chem., 2004, 76, 6247-6253.

16 D. R. Gossett, W. M. Weaver, A. J. Mach, S. C. Hur, H. T. Tse, W. Lee, H. Amini and D. Di Carlo, Anal. Bioanal. Chem., 2010, 397, 3249-3267.

17 W. H. Coulter, Proc. Natl. Electron. Conf., 1956, 12, 1034-1040.

18 T. Sun and H. Morgan, Microfluid. Nanofluid., 2010, 8, 423-443.

19 D. Holmes, D. Pettigrew, C. Reccius, J. D. Gwyer, C. van Berkel, J. Holloway, D. E Davies and H. Morgan, Lab Chip, 2009, 9, 28812889.

20 D. Holmes and H. Morgan, Anal. Chem., 2010, 82, 1455-1461.

21 K. C. Cheung, M. Di Berardino, G. Schade-Kampmann, M. Hebeisen, A. Pierzchalski, J. Bocsi, A. Mittag and A. Tarnok, Cytometry, Part A, 2010, 77A, 648-666.

22 R. M. Pugo, S. C. Deane, C. Glasse, M. R. Burcher, H. Morgan and C. H. Reccius, Flow speed particle focussing in microfluidic impedance measurements, in Micro Total Analysis Systems, Groningen, Netherlands, 2010.

23 S. L. Ledis, H. R. Crews, T. J. Fischer and T. Sena, Lysing reagent system for isolation, identification and/or analysis of leukocytes from whole bloodsamples, US5155044, 1992.

24 T. Sun, C. van Berkel, N. G. Green and H. Morgan, Microfluid. Nanofluid., 2009, 6, 179-187.

25 J. B. Bain, Blood Cells: A Practical Guide, Blackwell, Oxford, 2006.

26 International Council for Standardization in Haematology, J. Clin. Pathol., 1982, 35, 1320-1322.

27 B. Furie, J. Zwicker, T. LaRocca, C. Kos, B. Bauer and B. C. Furie, Haematol. Rep., 2005, 9, 5-8. 